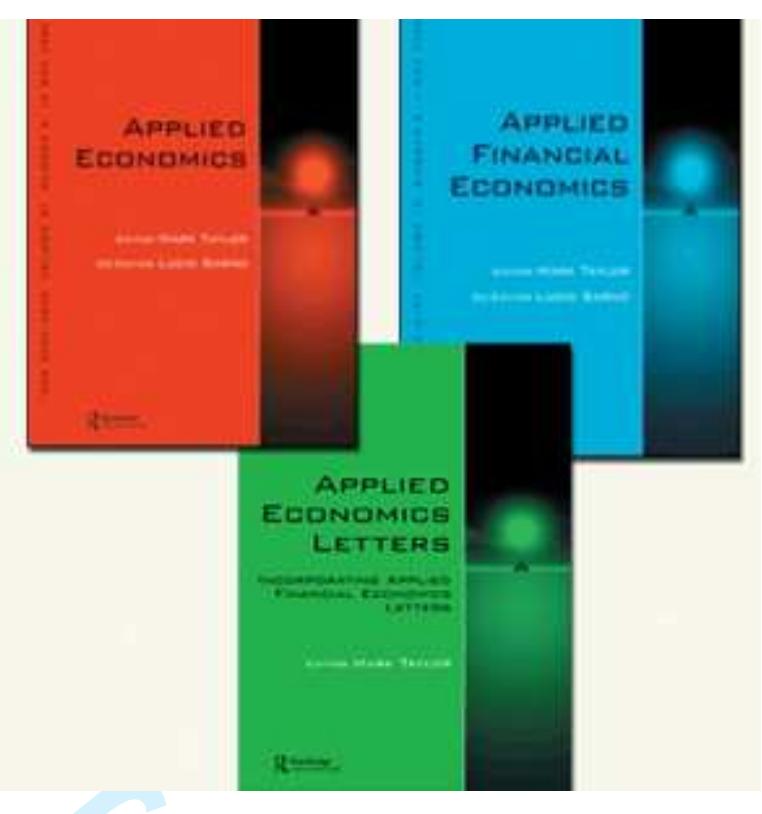

\title{
Examining the power of stochastic unit root tests without assuming independence in the error processes of the underlying time series
}

\begin{tabular}{|c|c|}
\hline Journal: & Applied Economics \\
\hline Manuscript ID: & Draft \\
\hline Journal Selection: & $\begin{array}{l}\text { Applied Economics Letters incorporating Applied Financial } \\
\text { Economics Letters }\end{array}$ \\
\hline $\begin{array}{r}\text { Date Submitted by the } \\
\text { Author: }\end{array}$ & $\mathrm{n} / \mathrm{a}$ \\
\hline Complete List of Authors: & Su, Jen-Je; Griffith University, Accounting, Finance and Economics \\
\hline JEL Code: & $\begin{array}{l}\text { C30 - General < C3 - Econometric Methods: Multiple/Simultaneous } \\
\text { Equation Models < C - Mathematical and Quantitative Methods, C32 } \\
\text { - Time-Series Models < C3 - Econometric Methods: } \\
\text { Multiple/Simultaneous Equation Models < C - Mathematical and } \\
\text { Quantitative Methods }\end{array}$ \\
\hline Keywords: & stochastic unit root, Monte Carlo simulation, test, power \\
\hline
\end{tabular}

\section{SCHOLARONE \\ Manuscripts}




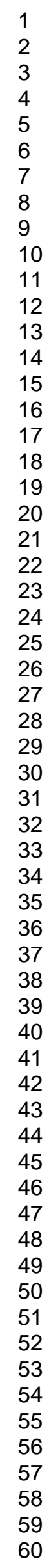

14

15

16

18

19

20

22

23

25

26

27

29

30

32

33

34

35

36

37

38

41

42

44

45

46

47

48

49

51

52

54

55

57

58

59

60

Editorial Office, Dept of Economics, Warwick University, Coventry CV4 7AL, UK 


\title{
Examining the power of stochastic unit root tests without assuming independence in the error processes of the underlying time series
}

\author{
Jen-Je Su* and Eduardo Roca \\ Department of Accounting, Finance and Economics \\ Griffith University \\ Nathan, Queensland, Australia
}

\begin{abstract}
Many studies have examined the power of stochastic unit root (STUR) tests. However, these studies assume that the two error processes of the underlying time series are independent. In this study, we undertake a Monte Carlo study on the power of STUR tests without the condition of independence among the error processes. The results show that the correlation between the two error processes may contribute profound impact to the power of STUR tests. Given the extensive use of STUR tests both as a diagnostic tool as well as a tool of analysis in economics and finance, this result therefore has very important implications for both theory and practice.
\end{abstract}

JEL Codes: C1, C22

Key Words: Stochastic Unit Root, Simulation, Power

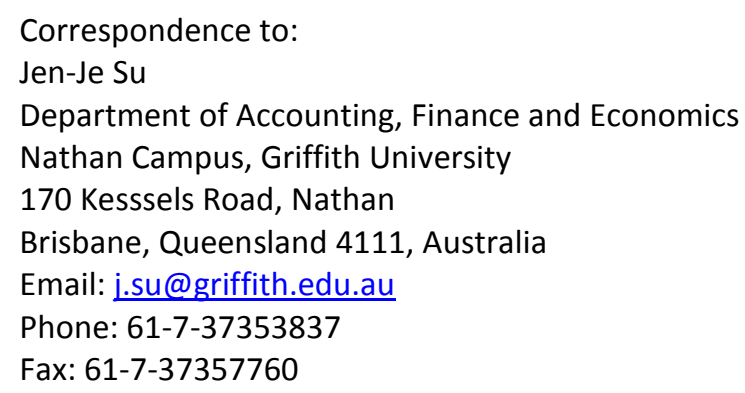




\section{INTRODUCTION}

Since the so-called "unit root revolution" of the 1980s, unit root tests have now been used extensively as a diagnostic tool in the selection of forecasting models (Phillips, 2009). Furthermore, in addition to serving as a diagnostic tool, the results of unit root tests have also been used as a direct tool for analysis of phenomena. However, it has long been argued that the conventional fixed coefficient unit-root model may not well represent many financial and macroeconomic time series - in particular, if the series are aggregated from different micro-level heterogeneous regimes. Abadir (2004) has showed that time-varying weights in aggregation would bring in heteroskedasticity and give rise to models with random coefficients. Specifically, the time-varying aggregation of autoregressive process may create a Random Coefficient Autoregressive (RCAR) model.

Consider the following RCAR model

$$
y_{t}=\left(\phi+\eta_{t}\right) y_{t-1}+\varepsilon_{t} \text { for } \mathrm{t}=1,2, \ldots
$$

where $\left(\eta_{t}, \varepsilon_{t}\right)^{\prime}$ is an i.i.d. random vector with $E\left(\varepsilon_{t}\right)=E\left(\eta_{t}\right)=0$, $\operatorname{var}\left(\varepsilon_{t}\right)=\sigma^{2}$, $\operatorname{var}\left(\eta_{t}\right)=\omega^{2}, \operatorname{cov}\left(\varepsilon_{t}, \eta_{t}\right)=\rho \omega$ and $|\rho| \leq \sigma$. This model is often referred as a standard (first-order) random coefficient autoregressive (RCAR(1)) model if the two error processes, the coefficient perturbation $\left(\eta_{t}\right)$ and the innovation $\left(\varepsilon_{t}\right)$, are not correlated $(\rho=0)$ and a generalized $\operatorname{RCAR}(1)$ model if $\rho \neq 0$. When $\omega^{2}=0$, the 
model in (1) becomes the usual $\mathrm{AR}(1)$ model. When $\phi=1$, the model contains a unit root and the root can be either fixed $\left(\omega^{2}=0\right)$ or stochastic $\left(\omega^{2} \neq 0\right)$.

In RCAR models, a key concern is determining whether the parameter is indeed constant (i.e. $\omega^{2}=0$ ) given that the model has a unit root - that is, if the unit root is fixed or stochastic [see McCabe and Tremayne (1995), Distaso (2008) and Nagakura (2009a)]. Interestingly, all the stochastic unit root (STUR) tests in the literature assume independence between the two error processes $\left(\eta_{t}\right.$ and $\left.\varepsilon_{t}\right)$ when examining the tests' finite-sample power. Thus, the power properties of the said tests without the independence assumption remain largely unknown. Given the observation that, in some economic/finance time series (such as GDP and interest rates), positive shocks $\left(\varepsilon_{t}>0\right)$ tend to be more persistent than negative ones, it is quite likely that $\eta_{t}$ and $\varepsilon_{t}$ are positively correlated (i.e. a positive/negative $\eta_{t}$ is more likely to associate with a positive/negative $\varepsilon_{t}$ ). With a positive/negative $\eta_{t}$, the realized AR coefficient $\left(\phi+\eta_{t}\right)$ becomes larger/smaller and the process more/less persistent. See Beaudry and Koop (1993) and Koenker and Xiao (2006).

In this paper, we contribute to the further development of STUR testing through a Monte Carlo study of the power of STUR tests that does not assume independence among the error processes of the underlying time series. Our simulation result shows that the impact of lifting the independence assumption is quite remarkable. The impact can be very dissimilar across the tests - some exhibit significant power 
gain while others tend to lose power. Also, there are cases where the power function is non-monotonic - the power may increase initially followed by a subsequent decrease (or vice versa) - as $\rho$ increases.

The rest of the paper is organized as follows. In Section 2, we review the LBI and LM tests. In Section 3, we report the simulation results. In Section 4, we summarize the findings.

\section{STOCHASTIC UNIT ROOT TESTS}

McCabe and Tremayne (1995) suggested a locally best invariant (LBI) test given the maintained unit-root assumption to determine if the root is fixed or a stochastic one. Lee (1998) derived a similar LBI test statistic assuming that the underlying model is stationary under both the null and alternative hypotheses. This test is then extended by Nagakura (2009a) to include both stationary and non-stationary cases. On the other hand, Distaso (2008) proposed a class of LM tests in a standard RCAR(1) context that include (i) a univariate test for parameter constancy (ii) a univariate test for a unit root, and (iii) a joint test for both. The aforementioned tests are defined as follows.

For observations $\left\{y_{t}\right\}_{t=0}^{T}$ from the RCAR(1) model defined in (1), McCabe and Tremayne (1995) propose an LBI test statistic to test if $\omega^{2}=0$, given that $\phi=1$, 


$$
L B I_{1}=\frac{T^{-3 / 2} \sum_{t=1}^{T} y_{t-1}^{2}\left(\left[\Delta y_{t}\right]^{2}-\hat{\sigma}_{\varepsilon}^{2}\right)}{\hat{\sigma}_{\varepsilon}^{2} \hat{\kappa}_{\varepsilon}}
$$

where $\Delta y_{t}=y_{t}-y_{t-1}, \hat{\kappa}_{\varepsilon}^{2}=T^{-1} \sum_{t=1}^{T}\left(\left[\Delta y_{t}\right]^{2}-\hat{\sigma}_{\varepsilon}^{2}\right)^{2}$ and $\hat{\sigma}_{\varepsilon}^{2}=T^{-1} \sum_{t=1}^{T}\left[\Delta y_{t}\right]^{2}$. The LBI test statistic of Lee (1998) and Nagakura (2009a) is derived under the same context with the assumption of $\phi=1$ being lifted and with $\phi$ consequently requiring estimation. The Lee and Nagakura's LBI test has the form

$$
L B I_{2}^{*}=\frac{\sum_{t=1}^{T} y_{t-1}^{2}\left(\left[y_{t}-\tilde{\phi} y_{t-1}\right]^{2}-\tilde{\sigma}_{\varepsilon}^{2}\right)}{\tilde{\kappa}_{\varepsilon}\left[\sum_{t=1}^{T}\left(y_{t-1}^{2}-\bar{y}_{-1}^{2}\right)^{2}\right]^{1 / 2}},
$$

where $\tilde{\phi}$ is the OLS estimate of $y_{t}$ on $y_{t-1}, \bar{y}_{-1}^{2}=T^{-1} \sum_{t=1}^{T} y_{t-1}^{2}, \tilde{\kappa}_{\varepsilon}^{2}=T^{-1} \sum_{t=1}^{T}\left(\tilde{\varepsilon}_{t}^{2}-\tilde{\sigma}_{\varepsilon}^{2}\right)^{2}$ and $\tilde{\sigma}_{\varepsilon}^{2}=T^{-1} \sum_{t=1}^{T} \tilde{\varepsilon}_{t}^{2}$ with $\tilde{\varepsilon}_{t}=y_{t}-\tilde{\phi} y_{t-1}$. The LM test of Distaso (2008) in this regard is

$$
L M^{*}=\frac{\left[\sum_{t=1}^{T} y_{t-1}^{2}\left(\left[y_{t}-\tilde{\phi} y_{t-1}\right]^{2}-\tilde{\sigma}_{\varepsilon}^{2}\right)\right]^{2}}{2 \tilde{\sigma}_{\varepsilon}^{2} \sum_{t=1}^{T} y_{t-1}^{4}\left(2\left[y_{t}-\tilde{\phi} y_{t-1}\right]^{2}-\tilde{\sigma}_{\varepsilon}^{2}\right)} .
$$

In this note, we focus on the maintained hypothesis that $\phi=1$; given that, the $\mathrm{LBI}_{2}$ test becomes

$$
L B I_{2}=\frac{\sum_{t=1}^{T} y_{t-1}^{2}\left(\left[\Delta y_{t}\right]^{2}-\hat{\sigma}_{\varepsilon}^{2}\right)}{\hat{\kappa}_{\varepsilon}\left[\sum_{t=1}^{T}\left(y_{t-1}^{2}-\bar{y}_{-1}^{2}\right)^{2}\right]^{1 / 2}},
$$

and the LM test becomes 


$$
L M=\frac{\left[\sum_{t=1}^{T} y_{t-1}^{2}\left(\left[\Delta y_{t}\right]^{2}-\hat{\sigma}_{\varepsilon}^{2}\right)\right]^{2}}{2 \hat{\sigma}_{\varepsilon}^{2} \sum_{t=1}^{T} y_{t-1}^{4}\left(2\left[\Delta y_{t}\right]^{2}-\hat{\sigma}_{\varepsilon}^{2}\right)}
$$

Under the null hypothesis that $\omega^{2}=0$ and with symmetric innovations in model (1) (i.e. $E\left(\varepsilon_{t}^{3}\right)=0$ ), the $\mathrm{LBI}_{2}$ test statistic follows the standard normal distribution asymptotically (Nagakura (Theorem1, 2009a)) while the $\mathrm{LBI}_{1}$ and LM statistics converge to some non-standard distributions (McCabe and Tremayne (Theorem 2, 1995) and Distaso (Theorem 1, 2008)) and their critical values are obtained through simulation. All three tests are right-tailed tests.

According to simulation results in McCabe and Tremayne (1995), McCabe and Smith (1998), Taylor and van Dijk (2002), Nagakura (2009a) and Distaso (2008), these three tests have finite sample power under the alternative hypothesis $\left(\omega^{2}>0\right)$ and the power increases as $\omega^{2}$ increases (with some exceptions for the $\mathrm{LBI}_{1}$ test). All of these Monte Carlo studies assume $\rho=0$.

\section{Simulation results when $\rho \neq 0$}

A Monte Carlo experiment was conducted according to model (1) given that $\phi=1$ and 


$$
\left(\begin{array}{l}
\varepsilon_{t} \\
\eta_{t}
\end{array}\right) \sim N I D\left(\left(\begin{array}{l}
0 \\
0
\end{array}\right),\left(\begin{array}{cc}
1 & \rho \omega \\
\rho \omega & \omega^{2}
\end{array}\right)\right) .
$$

Simulations are performed in GAUSS with 10,000 iterations with different values of the parameters $\left(\rho, \omega^{2}\right)$ and sample sizes $(T)$. The empirical rejection probabilities of the $\mathrm{LBI}_{1}, \mathrm{LBI}_{2}$ and $\mathrm{LM}$ tests are reported in Table 1 at the $5 \%$ significance level.

The results from Table 1 are summarized as follows. All three tests have correct size under the null $\left(\omega^{2}=0\right)$. When $\eta_{t}$ and $\varepsilon_{t}$ are independent $(\rho=0)$, the result is similar to the results of McCabe and Tremayne (1995), Distaso (2008) and Nagakura (2009a). While the power of the $\mathrm{LBI}_{2}$ and $\mathrm{LM}$ tests increases uniformly as $\omega^{2}$ increases, the $\mathrm{LBl}_{1}$ test seems not follow the same pattern - when $\mathrm{T}$ is large $(T=1000)$, the power of the $\mathrm{LBI}_{1}$ test increases first but drops considerably later on. Thus, the $\mathrm{LBI}_{2}$ and $\mathrm{LM}$ tests are consistent but the $\mathrm{LBI}_{1}$ test is not - see Nagakura (2009b).

Allowing $\rho \neq 0$, the empirical power behavior of the tests becomes rather complicated. For the $\mathrm{LBI}_{1}$ test, when $\rho$ increases, it tends to gain power when $\omega^{2}$ is very small (say, 0.0001) and loses power when $\omega^{2}$ gets larger (say, 0.01), while the power is invariant to $\rho$ (unless it is nearly one) when $\omega^{2}$ gets even larger (say, 0.1). These patterns appear quite stable across different sample sizes. Despite the $\mathrm{LBI}_{2}$ test possessing a power that is almost always higher than the $\mathrm{LBI}_{1}$ test, the two tests share quite similar power pattern concerning $\rho$. On the other hand, as $\rho$ increases, 
the empirical power of the LM test increases monotonically when $\omega^{2}$ is small, decreases monotonically when $\omega^{2}$ get larger but the power becomes invariant to $\rho$ (unless it is nearly one) when $\omega^{2}$ gets even larger, and decreases initially followed by a subsequent increase when $\omega^{2}$ is moderate. Like the LBI tests, the pattern is stable across different sample sizes.

We also plot the power function against $\rho$ (from 0 to 1 with a grid of 0.05 ) for $\mathrm{T}=500$ in Figure 1 . With $\omega^{2}$ equal to 0.0001 , all three tests gain power as $\rho$ increases with the LM test enjoying the largest gain among the three. With $\omega^{2}$ equal to 0.001 and 0.01 , both LBI tests lose power, while the LM test first loses but then gains power as $\rho$ increases. With $\omega^{2}$ equal to 0.1 , the three tests are insensitive to $\rho$ if $\rho$ is not too close to unity and they all drop in power considerably when $\rho$ is almost one.

\section{CONCLUSION}

This note examines the finite-sample power properties of tests for a fixed unit root of a time series against the stochastic unit root alternative. It has been shown that the correlation between the coefficient perturbations and the innovations of the underlying stochastic unit root process can have remarkable impact on the power of these tests. 


\section{REFERENCES}

Abadir, K.M. (2004) Cointegration theory, equilibrium and disequilibrium economics, The Manchester School, 72, 60-71.

Beaudry, P., Koop, G. (1993) Do Recessions Permanently Change Output? Journal of Monetary Economics, 31, 149-163.

Distaso, W. (2008) Testing for unit root processes in random coefficient autoregressive models, Journal of Econometrics, 142, 581-609.

Knoeker, R. Xiao, Z. (2006) Quantile Autoregression. Journal of the American Statistical Association, 101, 980-990.

Lee, S. (1998) Coefficient constancy test in a random coefficient autoregressive Model, Journal of Statistical Planning and Inference, 74, 93-101.

Leybourne, S.J., McCabe, B.P., Tremayne, A.R. (1996) Can economic time series be differenced to stationary? Journal of Business and Economic Statistics, 14, 435446.

McCabe, B.P.M., Smith, R. (1998) The power of some tests for difference stationarity under local heteroscedatic integration, Journal of the American Statistical Association, 93, 751-761.

McCabe, B.P.M., Tremayne, A.R. (1995) Testing a time series for difference stationarity, The Annals of Statistics, 23, 1015-1028.

Nagakura, A. (2009a). Testing for coefficient stability of AR(1) model when the null is an integrated or a stationary process, Journal of Statistical Planning and Inference, 139, 2731-2745.

Nagakura, A. (2009b) Asymptotic theory for explosive random coefficient autoregressive models and inconsistency of a unit root test against a stochastic unit root process, Statistics and Probability Letters, 24, 2476-2483.

Phillips, P.C.B. (2009) Econometric theory and practice, Econometric Theory, 25, 583586.

Taylor, A.M, van Dijk, D. (2002) Can tests for stochastic unit roots provide useful portmanteau tests for persistence? Oxford Bulletin of Economics and Statistics, 64, 381-397. 
Table 1. Empirical Power of the $\mathrm{LM}$ and $\mathrm{LBI}_{1}$ and $\mathrm{LBI}_{2}$ tests

\begin{tabular}{|c|c|c|c|c|c|c|c|c|c|c|}
\hline \multirow[b]{2}{*}{$\rho$} & \multirow[b]{2}{*}{$\omega^{2}$} & \multicolumn{3}{|c|}{$T=100$} & \multicolumn{3}{|c|}{$\mathrm{T}=500$} & \multicolumn{3}{|c|}{$\mathrm{T}=1000$} \\
\hline & & $\mathrm{LBI}_{1}$ & $\mathrm{LBI}_{2}$ & LM & $\mathrm{LBI}_{1}$ & $\mathrm{LBI}_{2}$ & LM & $\mathrm{LBI}_{1}$ & $\mathrm{LBI}_{2}$ & LM \\
\hline 0 & 0 & 0.053 & 0.053 & 0.052 & 0.050 & 0.047 & 0.049 & 0.050 & 0.050 & 0.050 \\
\hline \multirow[t]{4}{*}{0} & 0.0001 & 0.060 & 0.049 & 0.045 & 0.117 & 0.106 & 0.080 & 0.227 & 0.265 & 0.211 \\
\hline & 0.001 & 0.105 & 0.090 & 0.080 & 0.411 & 0.619 & 0.559 & 0.603 & 0.903 & 0.891 \\
\hline & 0.01 & 0.351 & 0.515 & 0.526 & 0.723 & 0.995 & 0.993 & 0.842 & 1.000 & 1.000 \\
\hline & 0.1 & 0.461 & 0.969 & 0.980 & 0.536 & 1.000 & 1.000 & 0.539 & 1.000 & 1.000 \\
\hline \multirow[t]{4}{*}{0.25} & 0.0001 & 0.053 & 0.050 & 0.051 & 0.118 & 0.135 & 0.114 & 0.206 & 0.282 & 0.252 \\
\hline & 0.001 & 0.110 & 0.098 & 0.084 & 0.386 & 0.559 & 0.514 & 0.578 & 0.817 & 0.794 \\
\hline & 0.01 & 0.338 & 0.497 & 0.454 & 0.706 & 0.985 & 0.982 & 0.823 & 1.000 & 1.000 \\
\hline & 0.1 & 0.464 & 0.958 & 0.956 & 0.531 & 1.000 & 1.000 & 0.542 & 1.000 & 1.000 \\
\hline \multirow[t]{4}{*}{0.5} & 0.0001 & 0.059 & 0.055 & 0.054 & 0.134 & 0.187 & 0.210 & 0.215 & 0.341 & 0.448 \\
\hline & 0.001 & 0.107 & 0.110 & 0.107 & 0.323 & 0.463 & 0.516 & 0.490 & 0.631 & 0.681 \\
\hline & 0.01 & 0.298 & 0.425 & 0.403 & 0.678 & 0.933 & 0.925 & 0.804 & 0.994 & 0.993 \\
\hline & 0.1 & 0.452 & 0.930 & 0.925 & 0.531 & 1.000 & 1.000 & 0.551 & 1.000 & 1.000 \\
\hline \multirow[t]{4}{*}{0.75} & 0.0001 & 0.060 & 0.058 & 0.060 & 0.166 & 0.246 & 0.363 & 0.243 & 0.389 & 0.707 \\
\hline & 0.001 & 0.113 & 0.134 & 0.162 & 0.276 & 0.419 & 0.791 & 0.390 & 0.490 & 0.865 \\
\hline & 0.01 & 0.236 & 0.370 & 0.434 & 0.579 & 0.763 & 0.818 & 0.740 & 0.924 & 0.938 \\
\hline & 0.1 & 0.438 & 0.846 & 0.838 & 0.536 & 0.999 & 0.999 & 0.543 & 1.000 & 1.000 \\
\hline \multirow[t]{4}{*}{0.9} & 0.0001 & 0.059 & 0.064 & 0.066 & 0.173 & 0.266 & 0.454 & 0.253 & 0.404 & 0.801 \\
\hline & 0.001 & 0.117 & 0.156 & 0.219 & 0.281 & 0.423 & 0.892 & 0.330 & 0.423 & 0.945 \\
\hline & 0.01 & 0.202 & 0.347 & 0.620 & 0.455 & 0.579 & 0.837 & 0.614 & 0.755 & 0.885 \\
\hline & 0.1 & 0.390 & 0.704 & 0.720 & 0.526 & 0.989 & 0.988 & 0.551 & 1.000 & 0.999 \\
\hline \multirow[t]{4}{*}{0.99} & 0.0001 & 0.067 & 0.066 & 0.071 & 0.186 & 0.296 & 0.512 & 0.255 & 0.406 & 0.824 \\
\hline & 0.001 & 0.135 & 0.166 & 0.262 & 0.278 & 0.415 & 0.915 & 0.324 & 0.413 & 0.965 \\
\hline & 0.01 & 0.199 & 0.342 & 0.676 & 0.291 & 0.387 & 0.927 & 0.359 & 0.439 & 0.916 \\
\hline & 0.1 & 0.239 & 0.438 & 0.610 & 0.458 & 0.815 & 0.873 & 0.531 & 0.948 & 0.964 \\
\hline
\end{tabular}


Figure 1: The power function of the $\mathrm{LM}, \mathrm{LBI}_{1}, \mathrm{LBI}_{2}$ tests against $\rho(\mathrm{T}=500)$

(a) $\omega^{2}=0.0001$

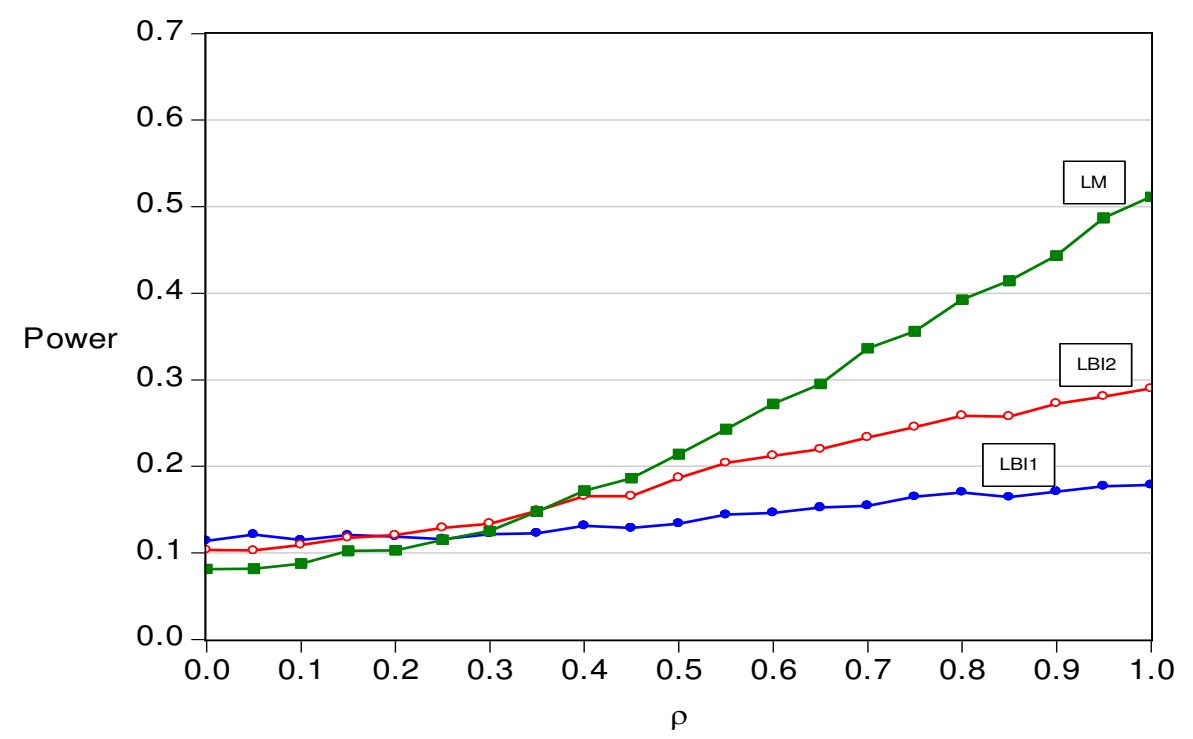

(b) $\omega^{2}=0.001$

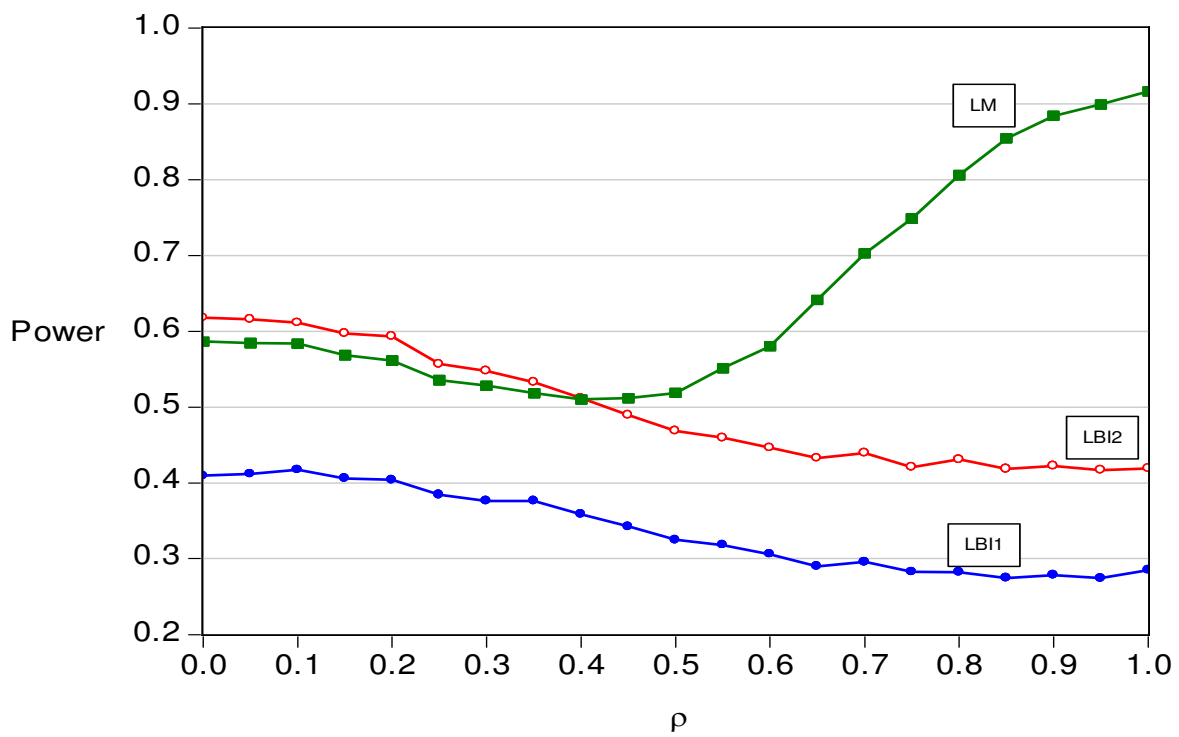


(c) $\omega^{2}=0.01$

1

2

3

4

5

6

10

11

12

13

14

15

16

17

18

19

20

21

22

23

24

25

26

27

28

29

30

31

32

33

34

35

36

37

38

39

40

41

42

43

44

45

46

47

48

49

50

51

52

53

54

55

56

57

58

59

60

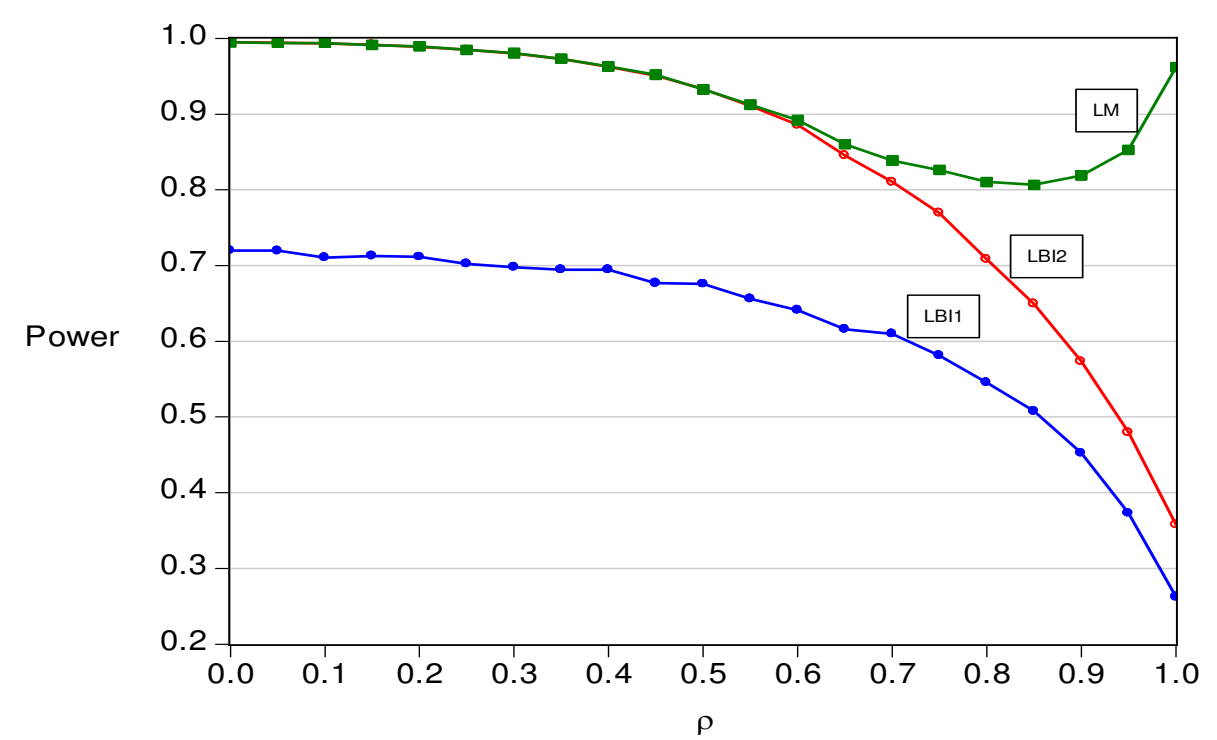

(d) $\omega^{2}=0.1$

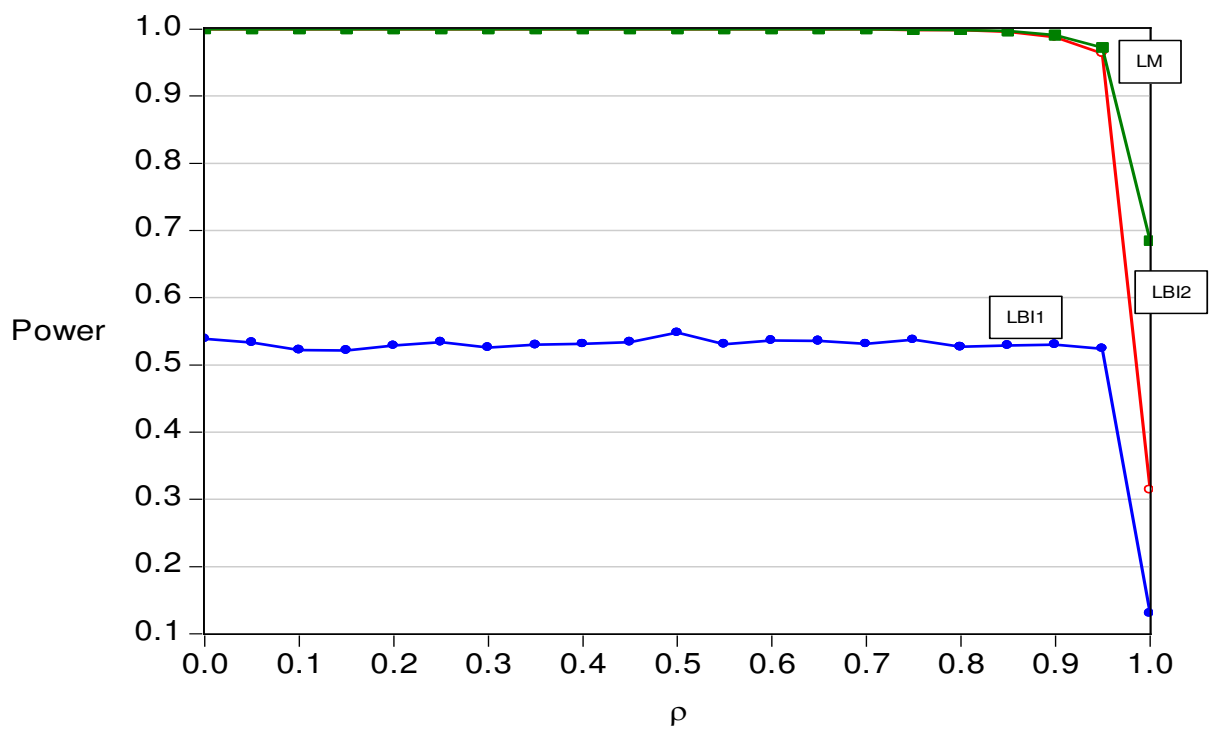

Editorial Office, Dept of Economics, Warwick University, Coventry CV4 7AL, UK 